\title{
PAPER \\ Maximum Likelihood Approach for RFID Tag Cardinality Estimation under Capture Effect and Detection Errors*
}

\author{
Chuyen T. NGUYEN ${ }^{\dagger a)}$, Nonmember, Kazunori HAYASHI ${ }^{\dagger b)}$, Megumi KANEKO $^{\dagger c}{ }^{\dagger}$ Members $^{\text {, }}$ \\ and Hideaki SAKAI ${ }^{\dagger \mathrm{d})}$, Fellow
}

\begin{abstract}
SUMMARY Cardinality estimation schemes of Radio Frequency IDentification (RFID) tags using Framed Slotted ALOHA (FSA) based protocol are studied in this paper. Not as same as previous estimation schemes, we consider tag cardinality estimation problem under not only detection errors but also capture effect, where a tag's IDentity (ID) might not be detected even in a singleton slot, while it might be identified even in a collision slot due to the fading of wireless channels. Maximum Likelihood (ML) approach is utilized for the estimation of the detection error probability, the capture effect probability, and the tag cardinality. The performance of the proposed method is evaluated under different system parameters via computer simulations to show the method's effectiveness comparing to other conventional approaches.

key words: RFID, Maximum Likelihood (ML), tag cardinality estimation, capture effect, detection errors
\end{abstract}

\section{Introduction}

Radio Frequency IDentification (RFID) technology is being increasingly deployed in many automated identification systems, such as inventory control and tracking, supply chain management and security check [1]-[4]. Tag cardinality estimation, where an RFID reader tries to estimate the total number of RFID tags in its communication range rapidly and reliably, is one of the fundamental tasks in RFID systems [5]. We can see the task in different applications such as Intelligent Transportation Systems (ITS) and indoor stadium systems [6] that track population distribution of vehicles and monitor visitors, respectively. The task can also be seen in other indoor applications such as factories or warehouses that store a huge number of products with the same type of identities, or in conferences, where thousands of participants need to be monitored [6], [7].

The Framed Slotted ALOHA (FSA) [8] based protocol is widely used in many RFID systems for tag cardinality estimation due to its simplicity and robustness [9]. In the protocol, the reader starts a reading round by first broadcasts a request message, which is a frame composed of time slots. Then, each tag responds to the reader in a randomly selected slot with its IDentity (ID). A slot is empty or sin-

\footnotetext{
Manuscript received September 5, 2012.

Manuscript revised December 21, 2012.

${ }^{\dagger}$ The authors are with the Graduate School of Informatics, Kyoto University, Kyoto-shi, 606-8501 Japan.

*This paper was presented in part at APWCS 2012 [21].

a) E-mail: ntchuyen@sys.i.kyoto-u.ac.jp

b)E-mail: kazunori@i.kyoto-u.ac.jp

c) E-mail: meg@i.kyoto-u.ac.jp

d)E-mail: hsakai@i.kyoto-u.ac.jp

DOI: 10.1587/transcom.E96.B.1122
}

gleton if no tag or only one tag responds to the reader in the slot, respectively. Otherwise, the slot is in collision. Here, the empty, singleton, collision slots could be distinguished by the reader. Indeed, in the case where an ID signal is detected, the reader confirms the correctness of the ID by verifying the Cyclic Redundancy Check (CRC) code carried in the ID, and if the code is valid, the slot, in which the ID is detected, is considered to be a singleton slot, otherwise, it might be an empty slot or a collision slot, which could be distinguished based on the received signal power of the slot: if the power is higher than a certain threshold then the slot is judged as a collision slot, otherwise as an empty slot. At the end of the reading round, the numbers of detected empty, singleton and collision slots are used for tag cardinality estimation, as adopted in several works [10]-[13].

All the above works, however, only consider tag cardinality estimation in a simple collision channel model, where the success of the detection of a tag solely depends on the status of the slot (empty, singleton or collision) in which the tag responds. The model is not reasonable in practice because the effect of wireless channels on the tag reading is ignored. Indeed, it is shown in [5] that an European Union tag operating at $868 \mathrm{MHz}$ requires 50 microwatts to respond from a distance of about 3.25 meters, while the required reader's sensitivity is 2500 mircowatts, under ideal conditions. Therefore, if we consider, for example, the fading phenomenon, the reader might not detect a tag's response even in a singleton slot if the received Signal-toNoise Ratio (SNR) is below the reader's sensitivity threshold, which we call "detection errors". Moreover, the reader might detect a tag's response even in a collision slot, which is called the capture effect [14]-[20], if the received Signalto-Interference plus Noise Ratio (SINR) is higher than the threshold due to the fading effect. In this case, the detected numbers of empty, singleton and collision slots no longer reflect the actual ones, which results in inaccurate tag cardinality estimation. Hence, effects of wireless channels such as the detection errors and the capture effect should be taken into consideration in tag cardinality estimation schemes.

Recently, Bo Li et al. [20] have proposed a Captureaware Minimum Error Backlog Estimation (CMEBE) method, in which a tag in a collision slot is assumed to be detected with a certain probability, which is called capture effect probability. Then, both the tag cardinality and the capture effect probability are estimated by minimizing the distance between the observed and expected vectors composed 
by the number of empty, singleton and collision slots. However, in CMEBE, the capture effect has not been studied in the context of fading, and hence the detection errors, which are also caused by wireless channels as mentioned above, have not been considered.

In this paper, we study tag cardinality estimation method for indoor RFID applications using FSA based protocol under not only the capture effect but also the detection errors in order to cope with the practical wireless environments with non-line-of-sight transmissions. Based on the assumption that the success of the detection of a tag in a slot depends on the received SNR or SINR, a tag may be undetected in a singleton slot with a certain probability (detection error probability), while it might be detected in a collision slot with another probability (capture effect probability). Maximum Likelihood (ML) approach is used for the estimation of the tag cardinality, the detection error probability and the capture effect probability. In particular, our considered system is modeled with flat Rayleigh fading channels, which have been shown, via measurements in [22]-[24], to be valid for indoor RFID systems with nonline-of-sight environments. The flat Rayleigh fading channel is also assumed in some other indoor RFID applications, which can be seen in [25]-[27]. Then, by assuming a certain SNR/SINR sensitivity threshold, the detection error and the capture effect probabilities are derived as a function of the threshold, which enables us to obtain the deterministic relation between the two probabilities. After that, since it is difficult to obtain the closed form solution of the ML problem, we utilize the steepest descent algorithm [28] based on the continuous relaxation of the tag cardinality. Computer simulations are performed to show the effectiveness of the proposed method comparing to the conventional CMEBE method.

The rest of this paper is organized as follows. In Sect. 2, the system model and the conventional approach CMEBE are described. Section 3 describes the proposed ML approach based on the analysis of detection error and capture effect probabilities. Simulation results are shown in Sect. 4, and finally, we conclude in Sect. 5.

\section{System Model and Conventional Approach}

\subsection{System Model}

The considered RFID system consists of a reader and $n$ tags as shown in Fig. 1. FSA based protocol is used in this paper with a fixed frame size $L$. Assuming flat Rayleigh fading channels, the received signal model at the reader in the $j$-th slot is described as

$$
y_{j}=\sum_{i \in \mathcal{K}_{j}} h_{i} x_{i}+w_{j}
$$

where $x_{i}$ is the transmitted signal from the $i$-th tag, while $\mathcal{K}_{j}$ is the set of indexes corresponding to the tags responding in the $j$-th slot. Here, if we denote by $\left|\mathcal{K}_{j}\right|$ the number of elements in $\mathcal{K}_{j}$, the $j$-th slot should be empty, singleton or

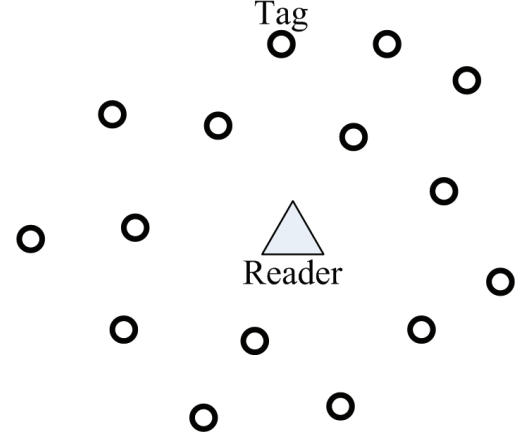

Fig. 1 System configuration.

collision when $\left|\mathcal{K}_{j}\right|=0,\left|\mathcal{K}_{j}\right|=1$ or $\left|\mathcal{K}_{j}\right| \geq 2$, respectively. $h_{i}$ is the fading coefficient between the $i$-th tag and the reader with $h_{i} \sim C \mathcal{N}(0,1)$. Note that in this paper, we ignore the effect of path-loss, which could be valid for indoor RFID applications, where the transmission is considered to be of short range [22], [23]. $w_{j}$ is an Additive White Gaussian Noise (AWGN) in the $j$-th slot with $w_{j} \sim \operatorname{CN}\left(0, \sigma^{2}\right)$.

Here, we assume that the reader detects a tag in a singleton slot only if the received SNR is greater than the reader's sensitivity threshold $\gamma$. Then, the detection error probability in the $j$-th slot, can be written as

$$
\beta_{j}=\beta=\operatorname{Pr}\left(\frac{\left|h_{i}\right|^{2}}{\sigma^{2}} \leq \gamma|| \mathcal{K}_{j} \mid=1, i \in \mathcal{K}_{j}\right) .
$$

Note that, because tags respond to the reader in randomly selected slots and path-loss is ignored, the detection error probability is supposed to be constant in every singleton slot for every tag. On the other hand, we also suppose that a tag might be detected in a collision slot if the received SINR is higher than $\gamma$. This means that several tags involved in the collision might be detected depending on the threshold. However, in common RFID systems, the reader finishes the tag detection process in each slot after detecting a valid ID, which implies that no more than one tag is detected in a slot. Hence, in the case of the capture effect, the received SINR of the detected tag is assumed to be not only higher than $\gamma$, but also the maximum among tags responding in the same slot. The capture effect probability in the $j$-th slot, which is denoted by $\alpha_{j}$, is thus written as

$$
\alpha_{j}=\operatorname{Pr}\left(\max _{i \in \mathcal{K}_{j}}\left\{\frac{\left|h_{i}\right|^{2}}{\sum_{k \in \mathcal{K}_{j}, k \neq i}\left|h_{k}\right|^{2}+\sigma^{2}}\right\}>\gamma|| \mathcal{K}_{j} \mid \geq 2\right) .
$$

We can see that if $\left|\mathcal{K}_{j}\right|$ is large, the capture effect probability is usually small because of the increased interference power. For simplicity, we restrict the capture effect in the collision slot to the case with $\left|\mathcal{K}_{j}\right|=2$ in the analysis, by which the capture effect probability is the same for all collision slots, and is denoted by $\alpha$. Thus, (3) could be rewritten with $\left|\mathcal{K}_{j}\right|=$ 2 as 


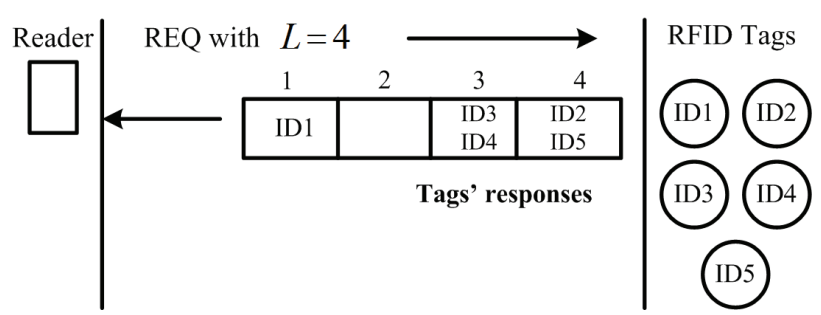

Fig. 2 Tags' responses.

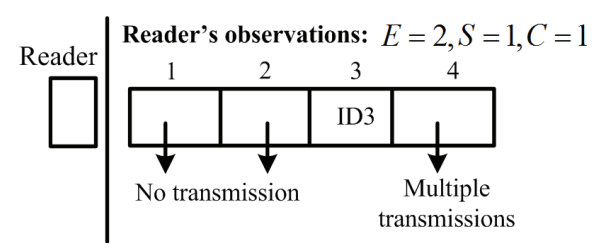

Fig. 3 Reader's observations.

$$
\alpha_{j} \approx \alpha=\operatorname{Pr}\left(\max \left\{\frac{\left|h_{i}\right|^{2}}{\left|h_{k}\right|^{2}+\sigma^{2}}, \frac{\left|h_{k}\right|^{2}}{\left|h_{i}\right|^{2}+\sigma^{2}}\right\}>\gamma|| \mathcal{K}_{j} \mid=2\right) .
$$

Note that the validity of this simplification is verified in the simulations.

After receiving the tags' responses, the reader distinguishes the status of a slot as follows. If a tag's ID is obtained correctly in the $j$-th slot, the slot is understood as a singleton slot by the reader. Otherwise, the reader treats the slot as an empty or a collision one if the received signal power is lower or higher than a certain threshold, respectively. In other words, if we denote by $\gamma_{C}$ the threshold, the undetected slot is judged as collision if

$$
\sum_{i \in \mathcal{K}_{j}}\left|h_{i}\right|^{2}+\sigma^{2}>\gamma_{C}
$$

otherwise as empty. Note that in (5), without loss of generality, the transmit power from the $i$-th tag is assumed to be 1. At the end of the reading round, the detected numbers of empty, singleton and collision slots are denoted as $E, S$ and $C$, respectively.

Figures 2 and 3 describe a simple example of our system model with a reading round, where the request $L=4$ is first transmitted to all tags, and each tag randomly selects one of 4 slots to respond (in Fig. 2). Due to the impact of wireless channels, tag 3 is detected in the collision slot 3 , while tag 1 is not observed in the singleton slot 1 . In this case, $E=2, S=1, C=1$ (in Fig. 3).

\subsection{Conventional Approach-CMEBE [20]}

In [20], although the detection error in a singleton slot is not considered, the capture effect probability is introduced with a fixed value for every collision slot. Under this assumption, the expected values of $E, S$ and $C$, which are denoted by $\bar{E}, \bar{S}$ and $\bar{C}$, respectively, are written as follows

$$
\begin{aligned}
& \bar{E}=L\left(1-\frac{1}{L}\right)^{n}, \\
& \bar{S}=n\left(1-\frac{1}{L}\right)^{n-1}+\alpha\left[L-L\left(1-\frac{1}{L}\right)^{n}-n\left(1-\frac{1}{L}\right)^{n-1}\right], \\
& \bar{C}=(1-\alpha)\left[L-L\left(1-\frac{1}{L}\right)^{n}-n\left(1-\frac{1}{L}\right)^{n-1}\right] .
\end{aligned}
$$

Then, the capture effect probability $\alpha$ and the total number of tags $n$ are estimated by minimizing the distance between the observed and theoretical reading results (similar to Vogt approach [11]) i.e.,

$$
\begin{aligned}
& \left(n_{\mathrm{CMEBE}}, \alpha_{\mathrm{CMEBE}}\right) \\
& =\arg \min _{n \in \mathbb{N}, \alpha \in[0,1]}\left\{(\bar{E}-E)^{2}+(\bar{S}-S)^{2}+(\bar{C}-C)^{2}\right\},
\end{aligned}
$$

where $n_{\mathrm{CMEBE}}$ and $\alpha_{\mathrm{CMEBE}}$ are the estimates of $n$ and $\alpha$, respectively. Here, for each of the possible values of $\alpha$, all possible values of $n$ are evaluated to find the solution that satisfies (7).

\section{Proposed Approach}

In this section, ML approach will be used to deal with tag cardinality estimation problem with the FSA based protocol, given the analysis of the detection error probability and the capture effect probability. In particular, we denote by $p_{0}, p_{1}$ or $p_{2}$ the probability that a given slot is empty, singleton or collision, respectively $\left(p_{0}+p_{1}+p_{2}=1\right)$, when $n$ tags respond to the reader in a time slotted frame size of $L$. $p_{0}, p_{1}, p_{2}$ are found in [13] as follows

$$
\begin{aligned}
& p_{0}=\left(1-\frac{1}{L}\right)^{n}, \\
& p_{1}=\frac{n}{L}\left(1-\frac{1}{L}\right)^{n-1}, \\
& p_{2}=1-p_{0}-p_{1} .
\end{aligned}
$$

Under the detection errors and the capture effect, the detected status of the slot (i.e., empty, singleton, or collision) by the reader might be different from the actual status. Thus, if we denote by $p_{E}, p_{S}$ or $p_{C}$ the probability that the $j$-th slot is detected as empty, singleton or collision, respectively, we have

$$
\begin{aligned}
& p_{E}=p_{0}+\beta p_{1}+(1-\alpha) p_{2} \operatorname{Pr}\left(\sum_{i \in \mathcal{K}_{j}}\left|h_{i}\right|^{2}+\sigma^{2} \leq \gamma_{C}\right), \\
& p_{S}=(1-\beta) p_{1}+\alpha p_{2}, \\
& p_{C}=(1-\alpha)\left(1-\operatorname{Pr}\left(\sum_{i \in \mathcal{K}_{j}}\left|h_{i}\right|^{2}+\sigma^{2} \leq \gamma_{C}\right)\right) p_{2} .
\end{aligned}
$$

Due to the diversity effects, the received signal power in a collision slot is supposed to be usually larger than the threshold $\gamma_{C}$, which implies that the probability a collision slot is 
detected by the reader as an empty one is very small. Thus, we assume

$$
\operatorname{Pr}\left(\sum_{i \in \mathcal{K}_{j}}\left|h_{i}\right|^{2}+\sigma^{2} \leq \gamma_{C}\right) \approx 0,
$$

which will be also verified in the simulations. Therefore, we could simplify $p_{E}, p_{S}, p_{C}$ by approximated formulas as follows

$$
\begin{aligned}
& p_{E} \approx p_{0}+\beta p_{1}, \\
& p_{S}=(1-\beta) p_{1}+\alpha p_{2}, \\
& p_{C} \approx(1-\alpha) p_{2} .
\end{aligned}
$$

If we denote by $\hat{n}, \hat{\alpha}$ and $\hat{\beta}$ the ML estimates of $n, \alpha$ and $\beta$, respectively, we have

$$
(\hat{n}, \hat{\alpha}, \hat{\beta})=\arg \max _{n \in \mathbb{N}, \alpha, \beta \in[0,1]} f(L, E, S, C \mid n, \alpha, \beta),
$$

where $f(L, E, S, C \mid n, \alpha, \beta)$ is the likelihood function of the number of tags $n$, the detection error probability $\beta$ and the capture effect probability $\alpha$, given the frame size $L$, the detected numbers of empty slots $E$, singleton slots $S$ and collision slots $C$ given by

$$
f(L, E, S, C \mid n, \alpha, \beta)=\frac{(E+S+C) !}{E ! S ! C !} p_{E}^{E} p_{S}^{S} p_{C}^{C} .
$$

Then, in order to maximize the likelihood function, we derive the detection error probability and the capture effect probability as functions of the threshold $\gamma$, assuming flat Rayleigh fading channels.

Because $h_{i} \sim C \mathcal{N}(0,1)$, the detection error probability in (2) is easily obtained as

$$
\beta=1-e^{-\sigma^{2} \gamma} \text {. }
$$

On the other hand, we denote the random variables $Z_{i}, Z_{k}$ and $Z$ as follows

$$
\begin{aligned}
& Z_{i}=\frac{\left|h_{i}\right|^{2}}{\left|h_{k}\right|^{2}+\sigma^{2}}, \\
& Z_{k}=\frac{\left|h_{k}\right|^{2}}{\left|h_{i}\right|^{2}+\sigma^{2}}, \\
& Z=\max \left\{Z_{i}, Z_{k}\right\} .
\end{aligned}
$$

(4) is thus rewritten as

$$
\alpha=\operatorname{Pr}\left(Z>\gamma|| \mathcal{K}_{j} \mid=2\right) .
$$

In order to determine $\alpha$, we find the probability density function (pdf) of $Z$, which is denoted by $f_{Z}(z)$. In particular, since $h_{i}, h_{k} \sim \mathcal{C N}(0,1)$, the pdfs of $Z_{i}$ and $Z_{k}$, which are denoted by $f_{Z_{i}}(z)$ and $f_{Z_{k}}(z)$ respectively, are found as

$$
\begin{aligned}
f_{Z_{i}}(z) & =f_{Z_{k}}(z)=e^{\sigma^{2}} \int_{0}^{\infty}\left(z^{\prime}+\sigma^{2}\right) e^{-\left(z^{\prime}+\sigma^{2}\right)(z+1)} d z^{\prime} \\
& =\frac{e^{-\sigma^{2} z}}{(z+1)^{2}}+\sigma^{2} \frac{e^{-\sigma^{2} z}}{(z+1)} .
\end{aligned}
$$

Note that, in (17), Eq. 3.351.3 in [30] has been used for the integrations. The cumulative distribution function (cdf) of $Z_{i}\left(Z_{k}\right)$ denoted by $F_{Z_{i}}(z)\left(F_{Z_{k}}(z)\right)$ is, then, written as

$$
\begin{aligned}
F_{Z_{i}}(z)=F_{Z_{k}}(z) & =\int_{0}^{z}\left(\frac{e^{-\sigma^{2} z^{\prime}}}{\left(z^{\prime}+1\right)^{2}}+\sigma^{2} \frac{e^{-\sigma^{2} z^{\prime}}}{\left(z^{\prime}+1\right)}\right) d z^{\prime} \\
& =1-\frac{e^{-\sigma^{2} z}}{z+1} .
\end{aligned}
$$

Thus, $f_{Z}(z)$ can be determined as in [31] as follows

$$
\begin{aligned}
f_{Z}(z) & =2 f_{Z_{i}}(z) F_{Z_{k}}(z) \\
& =2\left(1-\frac{e^{-\sigma^{2} z}}{z+1}\right)\left(\frac{e^{-\sigma^{2} z}}{(z+1)^{2}}+\sigma^{2} \frac{e^{-\sigma^{2} z}}{(z+1)}\right) .
\end{aligned}
$$

(16) is, therefore, rewritten as

$$
\begin{aligned}
\alpha= & \int_{\gamma}^{\infty}\left(\frac{2 e^{-\sigma^{2} z}}{(z+1)^{2}}+\frac{2 \sigma^{2} e^{-\sigma^{2} z}}{z+1}\right) d z \\
& -\int_{\gamma}^{\infty}\left(\frac{2 e^{-2 \sigma^{2} z}}{(z+1)^{3}}+\frac{2 \sigma^{2} e^{-2 \sigma^{2} z}}{(z+1)^{2}}\right) d z \\
= & \frac{2 e^{-\sigma^{2} \gamma}}{\gamma+1}-\frac{e^{-2 \sigma^{2} \gamma}}{(\gamma+1)^{2}}(\text { by using (14)) } \\
= & \frac{2 \sigma^{2}(1-\beta)}{\sigma^{2}-\ln (1-\beta)}-\left(\frac{\sigma^{2}(1-\beta)}{\sigma^{2}-\ln (1-\beta)}\right)^{2} .
\end{aligned}
$$

Since now we have a deterministic relation between $\alpha$ and $\beta$, by substituting $\alpha$ in (20) into (13), we can rewrite the likelihood function as a function of $\beta$ and $n$ as follows

$$
\begin{aligned}
& f(L, E, S, C \mid n, \beta)=\frac{(E+S+C) !}{E ! S ! C !}\left(p_{0}+\beta p_{1}\right)^{E} \times \\
& \left((1-\beta) p_{1}+\left(\frac{\sigma^{2}(1-\beta)\left(\sigma^{2}+\sigma^{2} \beta-2 \ln (1-\beta)\right)}{\left(\sigma^{2}-\ln (1-\beta)\right)^{2}}\right) p_{2}\right)^{S} \\
& \times\left(\left(\frac{\sigma^{2} \beta-\ln (1-\beta)}{\sigma^{2}-\ln (1-\beta)}\right)^{2} p_{2}\right)^{C} .
\end{aligned}
$$

Although we have simplified the likelihood function by reducing the number of variables from three to two, it is still difficult to obtain a closed form solution of the ML problem. In this paper, we utilize the steepest descent algorithm to find the optimal solution employing the continuous relaxation of $n$. In particular, we denote by $\hat{n}_{r}, \hat{\beta}_{r}$, and $\nabla f_{r}$ the estimates of $n, \beta$, and the gradient of the $\log$ likelihood function, respectively, in the $r^{\text {th }}$ round. The update equation is given by

$$
\left(\begin{array}{c}
\hat{n}_{r} \\
\hat{\beta}_{r}
\end{array}\right)=\left(\begin{array}{c}
\hat{n}_{r-1} \\
\hat{\beta}_{r-1}
\end{array}\right)+\xi \nabla f_{r-1},
$$

where $\xi$ is the step size, and $\nabla f_{r}$ is obtained as

$$
\nabla f_{r}=\left.\left(\begin{array}{c}
\frac{\partial \ln f(L, E, S, C \mid n, \beta)}{\partial n} \\
\frac{\partial \ln f(L, E, S, C \mid n, \beta)}{\partial \beta}
\end{array}\right)\right|_{n=\hat{n}_{r}, \beta=\hat{\beta}_{r}}
$$




$$
\begin{aligned}
& g_{2}(n, \beta)=\frac{E\left[(L-1) \ln \left(1-\frac{1}{L}\right)+\beta\left(1+n \ln \left(1-\frac{1}{L}\right)\right)\right]}{L-1+\beta n} \\
& +\frac{S\left[\frac{1-\beta}{L}\left(\left(1-\frac{1}{L}\right)^{n-1}+n\left(1-\frac{1}{L}\right)^{n-1} \ln \left(1-\frac{1}{L}\right)\right)+f_{1}(\beta)\left(-\left(1-\frac{1}{L}\right)^{n} \ln \left(1-\frac{1}{L}\right)-\frac{1}{L}\left(\left(1-\frac{1}{L}\right)^{n-1}+n\left(1-\frac{1}{L}\right)^{n-1} \ln \left(1-\frac{1}{L}\right)\right)\right)\right]}{(1-\beta) \frac{n}{L}\left(1-\frac{1}{L}\right)^{n-1}+f_{1}(\beta)\left[1-\left(1-\frac{1}{L}\right)^{n}-\frac{n}{L}\left(1-\frac{1}{L}\right)^{n-1}\right]} \\
& +C \frac{-\left(1-\frac{1}{L}\right)^{n} \ln \left(1-\frac{1}{L}\right)-\frac{1}{L}\left(\left(1-\frac{1}{L}\right)^{n-1}+n\left(1-\frac{1}{L}\right)^{n-1} \ln \left(1-\frac{1}{L}\right)\right)}{1-\left(1-\frac{1}{L}\right)^{n}-\frac{n}{L}\left(1-\frac{1}{L}\right)^{n-1}} .
\end{aligned}
$$

$$
=\left.\left(\begin{array}{l}
g_{1}(n, \beta) \\
g_{2}(n, \beta)
\end{array}\right)\right|_{n=\hat{n}_{r}, \beta=\hat{\beta}_{r}},
$$

where $g_{2}(n, \beta)$ can be seen from (24) in the next page, while, $g_{1}(n, \beta)$ is written as

$$
g_{1}(n, \beta)=\frac{E p_{1}}{p_{0}+\beta p_{1}}+\frac{S\left(-p_{1}+p_{2} f_{2}(\beta)\right)}{(1-\beta) p_{1}+f_{1}(\beta) p_{2}}-\frac{C f_{2}(\beta)}{1-f_{1}(\beta)},
$$

with

$$
\begin{aligned}
& f_{1}(\beta)=\frac{2 \sigma^{2}(1-\beta)}{\sigma^{2}-\ln (1-\beta)}-\left(\frac{\sigma^{2}(1-\beta)}{\sigma^{2}-\ln (1-\beta)}\right)^{2}, \\
& f_{2}(\beta)=\frac{2 \sigma^{2}\left[\ln (1-\beta)-\sigma^{2}-1\right]\left[\sigma^{2} \beta-\ln (1-\beta)\right]}{\left[\sigma^{2}-\ln (1-\beta)\right]^{3}} .
\end{aligned}
$$

The update process is repeated until

$$
\left|\ln f\left(L, E, S, C \mid \hat{n}_{r}, \hat{\beta}_{r}\right)-\ln f\left(L, E, S, C \mid \hat{n}_{r-1}, \hat{\beta}_{r-1}\right)\right|<\epsilon,
$$

where $\epsilon$ is a predetermined constant. Finally, $\hat{\alpha}$ is determined with (20) by substituting $\hat{\beta}_{r}$ into $\beta$.

\section{Simulation Results}

In this section, we show the performance of the proposed method under different system parameters via computer simulations. The performance of the method is also compared with that of CMEBE method. The values of $\xi$ and $\epsilon$ are set to be 0.01 and $10^{-4}$, respectively. The simulation results are obtained by Monte Carlo method with $R=10,000$ runs.

In order to support the validity of our assumption for the capture effect probability $\alpha$ in Sect. 2, we first plot $\alpha$ with respect to the number of tags in a collision slot $\left|\mathcal{K}_{j}\right|$. The variance of the AWGN $\left(\sigma^{2}\right)$ is set to be 0.1 (if not otherwise specified). In this figure, $\gamma$ is selected to be $8(\beta \approx 0.5$, based on (14)), which corresponds to the SNR of $20 \mathrm{~dB}$, while similar curves are obtained with other values of $\gamma$. We can see that because of the interference, $\alpha$ is decreased when $\left|\mathcal{K}_{j}\right|$ is

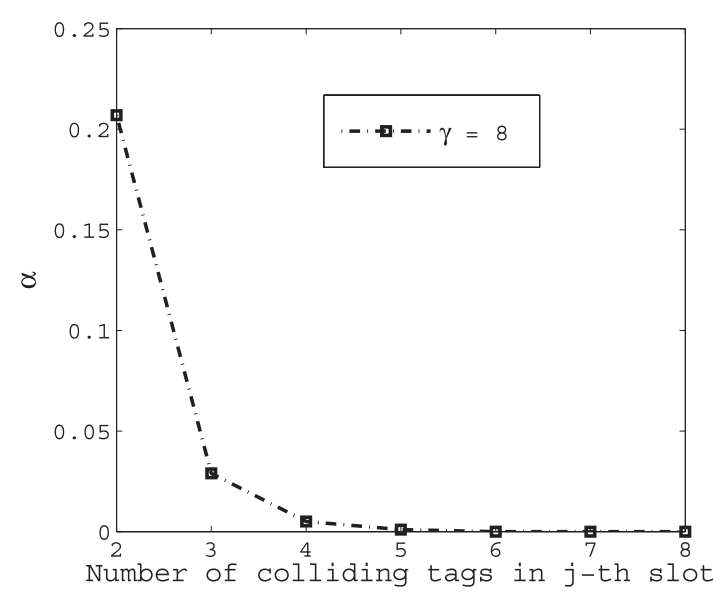

Fig. 4 Probability $\alpha$ w.r.t. $\left|\mathcal{K}_{j}\right|$.

Table $1 \operatorname{Pr}\left(\sum_{i \in \mathcal{K}_{j}}\left|h_{i}\right|^{2}+\sigma^{2}<\gamma_{C}|| \mathcal{K}_{j} \mid \geq 2\right)$.

\begin{tabular}{|l|l|}
\hline$\gamma_{C}$ & $\operatorname{Pr}\left(\sum_{i \in \mathcal{K}_{j}}\left|h_{i}\right|^{2}+\sigma^{2}<\gamma_{C}\left|\mathcal{K}_{j}\right| \geq 2\right)$ \\
\hline 0.4 & 0.0214 \\
\hline 0.3 & 0.0062 \\
\hline
\end{tabular}

increased, and if $\left|\mathcal{K}_{j}\right|>2, \alpha$ takes very small values compared as the case with $\left|\mathcal{K}_{j}\right|=2$, which matches with our assumption.

We also validate the assumption in (10) by showing the probability that a collision slot is judged as an empty slot $\operatorname{Pr}\left(\sum_{i \in \mathcal{K}_{j}}\left|h_{i}\right|^{2}+\sigma^{2}<\gamma_{C}|| \mathcal{K}_{j} \mid \geq 2\right)$ with different values of $\gamma_{C}$ in Table 1 , where we can see that the probability is very small.

In Fig. 5 we plot a typical example of the likelihood function $f(L, E, S, C \mid n, \beta)$ with respect to $n$ and $\beta$, in which the actual values of $n, L$ and $\beta$ are set to be 300,128 and 0.4 , respectively, when we have observed $E=40, S=48$ and $C=40$. Although it is difficult to verify the convergence of the proposed algorithm analytically, from the numerical example, we can expect the convergence of our algorithm to the true solution.

In Fig. 6, we plot the probabilities $\alpha$ and $\beta$ determined by CMEBE method and the proposed method with respect to $\gamma$, in which $L=256$ and $n=400$. Note that, plots with 


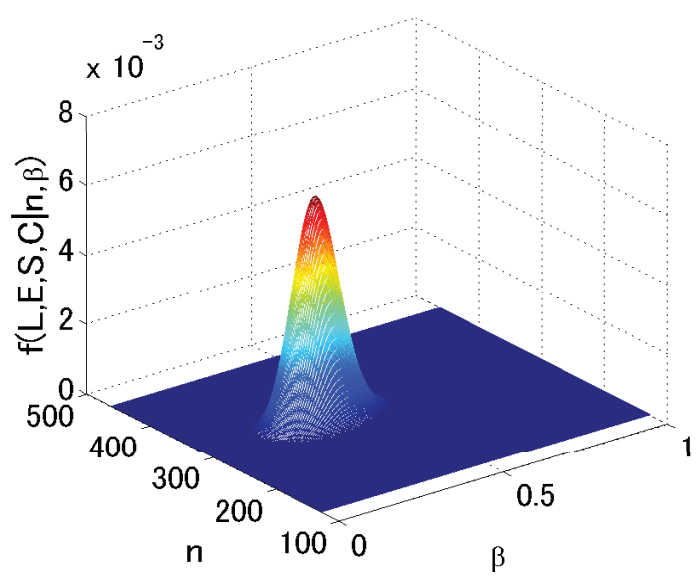

Fig. 5 Likelihood function $f(L, E, S, C \mid n, \beta)$ w.r.t. $n$ and $\beta$ (Peak: $n=$ $310, \beta=0.36$ ).

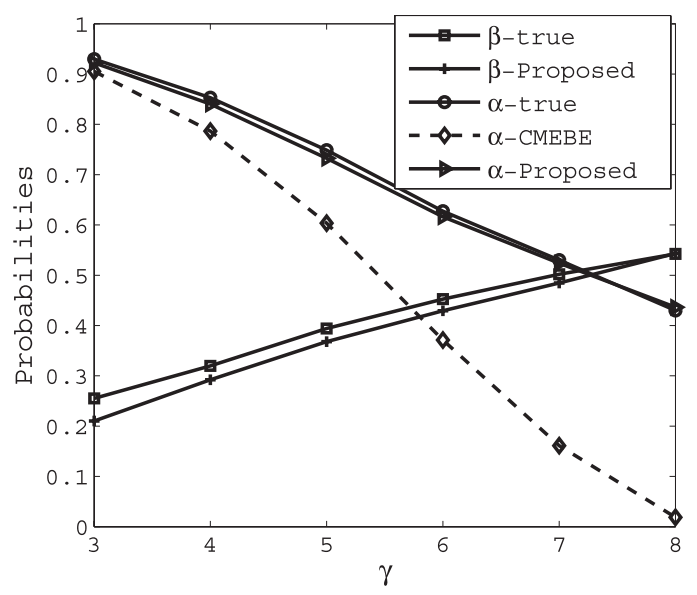

Fig. 6 Probabilities $\alpha$ and $\beta$ w.r.t. $\gamma$.

"true" are the true values of $\alpha$ and $\beta$ in the simulation. Also, the estimate of $\beta$ is plotted only for the proposed method since the detection errors are not considered in CMEBE method. We can see that the estimate of $\alpha$ obtained by the proposed method is very close to the true value, and is closer than that determined by CMEBE method. Thus, we can say that the proposed method shows a better performance than CMEBE method in terms of the estimated capture effect probability accuracy. This is because our method considers not only the capture effect but also the detection errors, utilizing ML approach. Note that, the estimates of $\beta$ and $\alpha$ obtained by the proposed method are underestimated comparing to the true values. This is because, we have assumed that the capture effect happens only with $\left|\mathcal{K}_{j}\right|=2$, while it actually happens when $\left|\mathcal{K}_{j}\right|>2$ as well, which we can see in Fig. 4. The underestimated $\alpha$ is compensated by decreasing the detection error probability $\beta$.

On the other hand, we also plot the probabilities $\alpha, \beta$ determined by CMEBE method and the proposed method with respect to $\sigma^{2}$ (or inverse SNR, in other words) in Fig. 7, and similarly to the previous case, the proposed method is observed to have quite accurate estimates of the probabilities

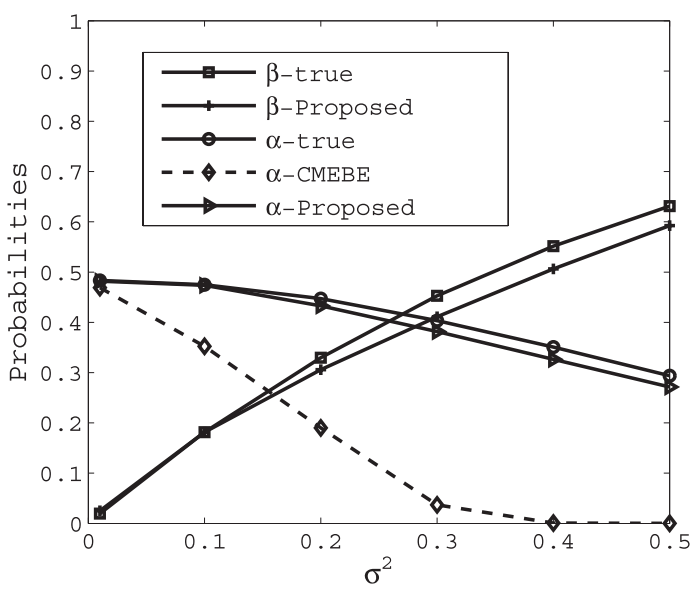

Fig. $7 \quad$ Probabilities $\alpha$ and $\beta$ w.r.t. $\sigma^{2}$.

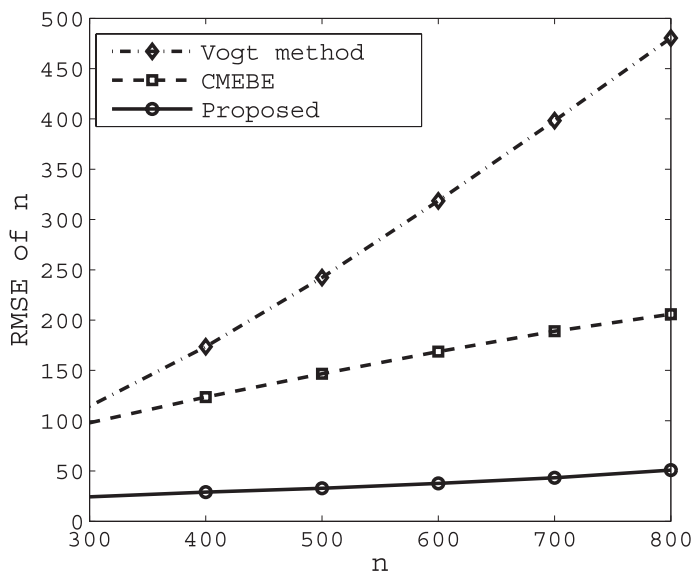

Fig. 8 RMSE of $n$ under capture effect and detection errors.

comparing to the true values, and the estimate of $\alpha$ is more accurate than that obtained by CMEBE method. Note that the performance of CMEBE method can be comparable as that of our method when $\sigma^{2} \approx 0$, because the SNR, in this case, is very high, and thus the detection error probability in each singleton slot is approximately equal to 0 .

We now study the effect of the detection errors and the capture effect to estimation schemes via the Root Mean Square Error (RMSE) performance of the estimated tag cardinality obtained by Vogt method [11], CMEBE method and the proposed ML approach in Fig. 8. $L$ and $n$ are, respectively, set to be 256 and from 300 to 800 . The RMSE of $n$ is defined as

$$
(\text { RMSE of } n)=\sqrt{\frac{1}{R} \sum_{i=1}^{R}\left(n_{i}-n\right)^{2}},
$$

where $n_{i}$ is the estimate of $n$ at the $i$-th simulation run. Note that, in Vogt method, neither the detection errors nor the capture effect is considered i.e., (6) and (7) are used with $\alpha=0$. We can see that the CMEBE method gives a more accurate estimate of $n$ than Vogt method, while our proposed method shows the best performance. This is because only the cap- 


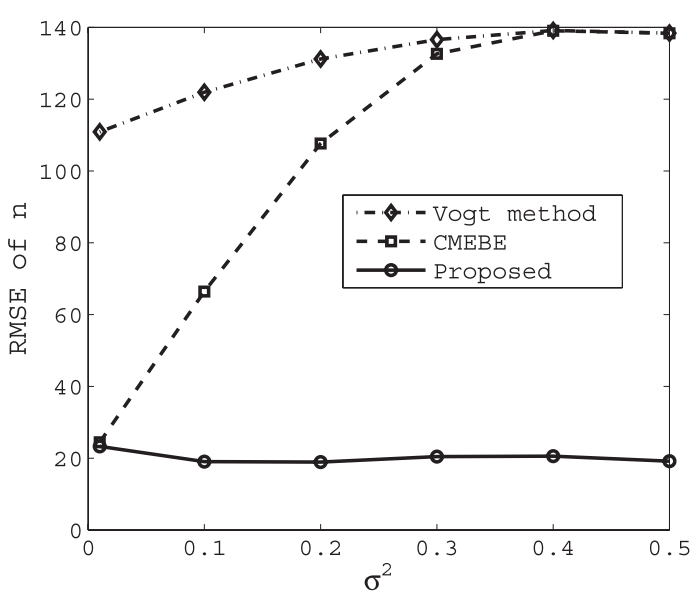

Fig. 9 RMSE of $n$ under capture effect and detection errors w.r.t. $\sigma^{2}$.

ture effect has been taken into account in CMEBE method, while our method deals with both phenomena, utilizing ML approach.

We now show the RMSE performance of the estimated tag cardinality obtained by all the above methods with different values of $\sigma^{2}$ in Fig. 9. The frame size $L$ and the actual number of tags $n$ are, respectively, set to be 256 and 400. We can see that when $\sigma^{2}$ is very small, which implies that the SNR is very high, almost all tags in singleton slots are detected successfully, by which the detection error probability is approximately equal to 0 (Fig. 7). Therefore, CMEBE method and the proposed method have the same performance and are better than Vogt method. However, when the noise power is increased, the detection error probability is also increased so that CMEBE method, which does not consider the detection error, is no longer effective as our method. On the other hand, as seen in Fig. 7, if the noise power keeps increasing, the estimate of $\alpha$ determined by CMEBE method is also not accurate, and is approximately equal to 0 . Hence, CMEBE method and Vogt method have the same performance, which can be seen in Fig. 9 with $\sigma^{2} \geq 0.4$.

\section{Conclusions}

In this paper, we studied tag cardinality estimation in RFID systems using ML approach and FSA based protocol in the context of fading wireless channels so that not only the capture effect but also the detection errors were considered. The relationship between detection error probability and the capture effect probability was derived assuming flat Rayleigh fading channels in order to simplify the likelihood function. We employed the steepest descent algorithm to find the estimates of the tag cardinality and the probabilities that maximize the likelihood function. Simulation results showed that our proposed method gave better performance than conventional methods, where the detection errors were not considered, in terms of the estimation accuracy.

In the future work, we intend to study RFID tag identification under both the detection errors and the capture ef- fect.

\section{References}

[1] K. Finkenzeller, RFID Handbook: Fundamentals and Applications in Contactless Smart Cards and Identification, Wiley \& Sons, 2003.

[2] R. Angeles, "RFID technologies: Supply-chain applications and implementations issues," Information Systems Management, vol.22, no.1, pp.51-65, 2005.

[3] A. Juels, D. Molnar, and D. Wagner, "Security and privacy issues in E-passports," Security and Privacy for Emerging Areas in Communications Networks, SecureComm, pp.74-88, 2005.

[4] D. Molnar and D. Wagner, "Privacy and security in library RFID: Issues, practices, and architectures," Proc. 11th ACM Conference on Computer and Communication Security, pp.210-219, 2004.

[5] M. Bolíc, D. Simplot-Ryl, and I. Stojmenovíc, RFID Systems: Research Trends and Challenges, Wiley \& Sons, 2010.

[6] C. Qian, H. Ngan, Y. Liu, and L.M. Ni, "Cardinality estimation for large-scale RFID systems," IEEE Trans. Parallel Distrib. Syst., vol.22, no.9, pp.1441-1454, 2011.

[7] C.T. Nguyen, K. Hayashi, M. Kaneko, P. Popovski, and H. Sakai, "Maximum likelihood approach for RFID tag set cardinality estimation with detection errors," J. Wirel. Pers. Commun., vol.67, no.4, pp.1-17, Dec. 2012.

[8] L.G. Roberts, "ALOHA packet system with and without slots and capture," ACM SIGCOMM Computer Communication Review, vol.5, pp.28-42, 1975.

[9] L. Zhu and T.-S.P. Yum, "The optimal reading strategy for EPC Gen2 RFID anti-collision systems," IEEE Trans. Commun., vol.58, no.9, pp.2725-2733, Sept. 2010.

[10] M. Kodialam and T. Nandagopal, "Fast and reliable estimation schemes in RFID systems," Proc. 12th Annual International Conference on Mobile Computing and Networking, pp.322-333, Sept. 2006.

[11] H. Vogt, "Efficient object identification with passive RFID tags," Proc. International Conference on Pervasive Computing, pp.98-113, 2002.

[12] F.C. Schoute, "Dynamic frame length ALOHA," IEEE Trans. Commun., vol.COM-31, no.4, pp.565-568, April 1983.

[13] W.-T. Chen, "An accurate tag estimate method for improving the performance of an RFID anticollision algorithm based on dynamic frame length ALOHA," IEEE Trans. Automation Science and Engineering, vol.6, no.1, pp.9-15, Jan. 2009.

[14] M. Victoria and Bueno-Delgado, "Analysis of the identification process in active RFID systems with capture effect," Proc. European Workshop on Smart Objects: Systems, Technologies and Applications, pp.1-6, 2010.

[15] J.E. Wieselthier, A. Ephremides, and L.A. Michaels, "An exact analysis and performance evaluation of framed ALOHA with capture," IEEE Trans. Wireless Commun., vol.37, no.2, pp.125-137, Feb. 1989.

[16] Y. Maguire and R. Pappu, "An optimal Q-algorithm for the ISO 18000-6C RFID protocol," IEEE Trans. Automation Science and Engineering, vol.6, no.1, pp.16-24, Jan. 2009.

[17] S.Y. Oh, S.H. Jung, J.W. Hong, and C.H. Lie, "A scheme to increase throughput in framed-ALOHA-based RFID systems with capture," ETRI Journal, vol.30, no.3, pp.486-488, June 2008.

[18] W.J. Shin and J.G. Kim, "A capture-aware access control method for enhanced RFID anti-collision performance," IEEE Commun. Lett., vol.13, no.5, pp.354-356, May 2009.

[19] Y.-C. Lai and L.-Y. Hsiao, "General binary tree protocol for coping with the capture effect in RFID tag identification," IEEE Commun. Lett., vol.14, no.3, pp.208-210, 2010.

[20] B. Li and J. Wang, "Efficient anti-collision algorithm utilizing the capture effect for ISO 18000-6C RFID protocol," IEEE Commun. Lett., vol.15, no.3, pp.352-354, 2011.

[21] C.T. Nguyen, K. Hayashi, M. Kaneko, and H. Sakai, "RFID tag car- 
dinality estimation under capture effect and detection errors," Proc. Asia Pacific Wireless Communications Symposium, Kyoto, Aug. 2012.

[22] M. Islam, S.A. Samad, M.A. Hannan, and A. Hussain, "Software defined radio for RFID signal in Rayleigh fading channel," Proc. IEEE Region 10 Conference TENCON, pp.1368-1372, Oct. 2010.

[23] M. Islam, M.A. Hanan, S.A. Samad, and A. Hussain, "Performance of RFID with AWGN and Rayleigh fading channels for SDR application," Proc. World Congress on Engineering, UK, July 2010.

[24] C. He and Z.J. Wang, "Closed-form BER analysis of non-coherent FSK in MISO double Rayleigh fading/RFID channel," IEEE Commun. Lett., vol.15, no.8, pp.848-850, Aug. 2011.

[25] S. Kai, H. Yigang, Z. Lei, and F. Gefeng, "Experimental and statistical analysis of blind spots for UHF RFID portal applications," Proc. 4th International Conferences on Electric Utility Deregulation and Restructuring and Power Technologies (DRPT), pp.1441-1444 China, July 2011.

[26] A. Lazaro, D. Girbau, and R. Villarino, "Effects of interferences in UHF RFID systems," Progress In Electromagnetics Research, PIER 98, pp.425-443, 2009.

[27] Z. Zhang, Z. Lu, Q. Chen, X. Yan, and L.-R. Zheng, "COSMO: $\mathrm{CO}$-simulation with MATLAB and OMNeT++ for indoor wireless networks," Proc. IEEE Globecom 2010, pp.1-6, 2010.

[28] J.F. Bonnans, J.C. Gilbert, C. Lemarecha, and C.A. Sagastizábal, Numerical Optimization: Theoretical and Practical Aspects, 2nd ed., Universitext, 2006

[29] "EPCglobal radio frequency identity protocols class-1 generation-2 UHF RFID protocol for communications at $860-960 \mathrm{MHz}$ version 1.2.0," Tech. Rep., 2008.

[30] I.S. Gradshteyn and I.M. Ryzhik, Table of Integrals, Series and Products (7th ed.), Academic Press, Elsevier, 2007.

[31] H. Stark and J.W. Woods, Probability and Random Processes with Applications to Signal Processing (3rd ed.), Prentice Hall, 2002.

[32] T.H. Cormen, C.E. Leiserson, R.L. Rivest, and C. Stein, Introduction to Algorithms, 3rd ed., MIT Press and McGraw-Hill, 2009.

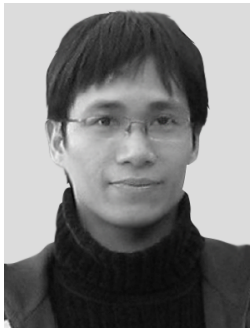

Chuyen T. Nguyen received his B.S. degree in Electronics and Telecommunications Engineering from Hanoi University of Technology, Hanoi, Vietnam and M.S. degree from Institute of Communications Engineering, National Tsing Hua University, Hsinchu, Taiwan, in 2006 and 2008, respectively. He is currently pursuing his Ph.D. degree in Graduate School of Informatics at Kyoto University, Japan. His research interests include statistics, optimization algorithms and their applications in wireless com-

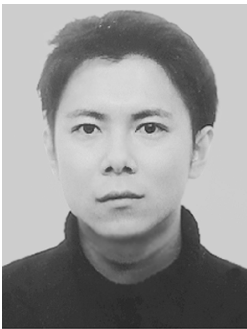

Kazunori Hayashi received the B.E., M.E and $\mathrm{Ph} . \mathrm{D}$. degrees in communication engineering from Osaka University, Osaka, Japan, in 1997, 1999 and 2002, respectively. Since 2002, he has been with the Department of Systems Science, Graduate School of Informatics, Kyoto University. He is currently an Associate Professor there. His research interests include digital signal processing for communication systems. He received the ICF Research Award from the KDDI Foundation in 2008, the IEEE Globecom 2009 Best Paper Award, the IEICE Communications Society Excellent Paper Award in 2011, the WPMC1 Best Paper Award, and the Telecommunications Advancement Foundation Award in 2012. He is a member of IEEE and ISCIE.

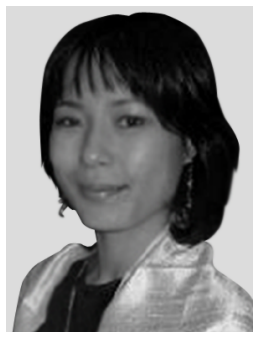

Megumi Kaneko received her B.S. and M.Sc. degrees in communication engineering in 2003 and 2004 from Institut National des Télécommunications (INT), France, jointly with an MSc. from Aalborg University, Denmark, where she received her Ph.D. degree in 2007. From January to July 2007, she was a visiting researcher in Kyoto University, Kyoto, Japan, and a JSPS post-doctoral fellow from April 2008 to August 2010. She is currently an Assistant Professor in the Department of Systems Science, Graduate School of Informatics, Kyoto University. Her research interests include wireless communication, protocol design and communication theory. She received the 2009 Ericsson Young Scientist Award, the IEEE Globecom 2009 Best Paper Award in Wireless Communications Symposium, the 2011 Funai Young Researcher's Award, the WPMC 2011 Best Paper Award and the 2012 Telecom System Technology Award.

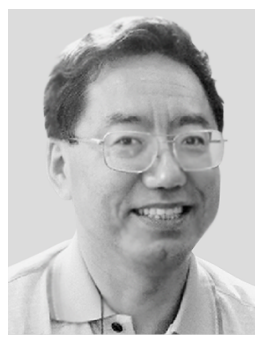

Hideaki Sakai received the B.E. and D.E degrees in applied mathematics and physics from Kyoto University, Kyoto, Japan, in 1972 and 1981, respectively. From 1975 to 1978 , he was with Tokushima University. He is currently a Professor in the Department of Systems Science, Graduate School of Informatics, Kyoto University. He spent 6 months from 1987 to 1988 at Stanford University as a Visiting Scholar. His research interests are in the areas of adaptive and statistical signal processing. $\mathrm{He}$ served as an associate editor of IEEE Transactions on Signal Processing from January 1999 to January 2001. He is a Fellow of the IEEE. 